\title{
When higher activations reflect lower deactivations: a PET study in Alzheimer's disease during encoding and retrieval in episodic memory
}

\author{
Alexandre Bejanin $1,2,3,4$, Armelle Viard ${ }^{1,2,3,4}{ }^{*}$, Gaël Chételat ${ }^{1,2,3,4}$, David Clarys ${ }^{5}$, Frédéric Bernard ${ }^{6}$, \\ Alice Pélerin ${ }^{1,2,3,4}$, Vincent de La Sayette ${ }^{1,2,3,4}$, Francis Eustache ${ }^{1,2,3,4}$ and Béatrice Desgranges ${ }^{1,2,3,4}$ \\ ${ }^{1}$ U1077, Inserm, Caen, France \\ 2 UMR-S1077, Université de Caen Basse-Normandie, Caen, France \\ ${ }^{3}$ UMR-S1077, Ecole Pratique des Hautes Etudes, Caen, France \\ ${ }^{4}$ U1077, Centre Hospitalier Universitaire, Caen, France \\ ${ }^{5}$ UMR-CNRS 6234, Centre de Recherches sur la Cognition et l'Apprentissage, Université de Poitiers, Poitiers, France \\ ${ }^{6}$ UMR 7237, Laboratoire d'Imagerie et de Neurosciences Cognitives, CNRS/Université de Strasbourg, Strasbourg, France
}

\section{Edited by:}

David Bartrés-Faz, University of

Barcelona, Spain

\section{Reviewed by:}

Rik Vandenberghe, Katholieke

Universiteit Leuven, Belgium

Lars Nyberg, Umeå University,

Sweden

\section{*Correspondence:}

Armelle Viard, U1077 GIP Cyceron, Inserm, Boulevard Henri Becquerel BP 5229, 14074 Caen Cedex, France. e-mail:viard@cyceron.fr
The aim of the present study was to explore the cerebral substrates of episodic memory disorders in Alzheimer's disease (AD) and investigate patients' hyperactivations frequently reported in the functional imaging literature. It remains unclear whether some of these hyperactivations reflect real increased activity or deactivation disturbances in the default mode network (DMN). Using positron emission tomography $\left({ }^{15} \mathrm{O}-\mathrm{H}_{2} \mathrm{O}\right)$, cerebral blood flow was measured in $11 \mathrm{AD}$ patients and 12 healthy elderly controls at rest and during encoding and stem-cued recall of verbal items. Subtractions analyses between the target and control conditions were performed and compared between groups. The average signal was extracted in regions showing hyperactivation in AD patients versus controls in both contrasts. To determine whether hyperactivations occurred in regions that were activated or deactivated during the memory tasks, we compared signal intensities between the target conditions versus rest. Our results showed reduced activation in AD patients compared to controls in several core episodic memory regions, including the medial temporal structures, during both encoding and retrieval. Patients also showed hyperactivations compared to controls in a set of brain areas. Further analyses conducted on the signal extracted in these areas indicated that most of these hyperactivations actually reflected a failure of deactivation. Indeed, whereas almost all of these regions were significantly more activated at rest than during the target conditions in controls, only one region presented a similar pattern of deactivation in patients. Altogether, our findings suggest that hyperactivations in AD must be interpreted with caution and may not systematically reflect increased activity. Although there has been evidence supporting the existence of genuine compensatory mechanisms, dysfunction within the DMN may be responsible for part of the apparent hyperactivations reported in the literature on AD.

Keywords: Alzheimer's disease, episodic memory, deactivation, medial temporal lobe, hippocampus, hyperactivation, compensatory mechanisms, functional imaging

\section{INTRODUCTION}

Episodic memory supports the encoding, storage, and retrieval of specific personally experienced events, situated in their spatiotemporal context of acquisition (Tulving, 1995). The impairment of this memory system often predominates in the clinical picture of Alzheimer's disease (AD) and is considered as a core diagnosis criterion (McKhann et al., 2011). Given the crucial role of the medial temporal lobe (MTL) in episodic memory, notably the hippocampus, the deficit of this memory system in $\mathrm{AD}$ is not surprising. Indeed, neuropathological abnormalities, especially neurofibrillary tangles, accumulate early in this region and induce a neuronal loss (Duyckaerts et al., 2009). Hence, the hippocampal region is regarded as one of the earliest cerebral structure affected by the pathological process (Braak and Braak, 1991) and its atrophy is part of the diagnosis biomarker support (McKhann et al., 2011). As pointed out by cognitivo-metabolic correlation studies (Desgranges et al., 1998; for review, see Salmon et al., 2008), the functional disturbance of this limbic structure is highly linked to the patients' episodic memory deficit. This approach has also highlighted other regions responsible for episodic memory deficits in the earlier stages of the disease, such as the posterior cingulate cortex (Chételat et al., 2003).

Task-related functional brain imaging techniques have been used to further investigate the substrate of episodic impairment in $\mathrm{AD}$. In a recent meta-analysis, comprising 14 functional imaging studies of episodic memory in AD, Schwindt and Black (2009) 
reported a bilateral hippocampal hypoactivation during information encoding in AD patients compared to elderly controls. During retrieval, no MTL activations were detected in the patient group, unlike the healthy elderly group. This MTL disturbance has been related to $\mathrm{AD}$ patients' weak memory performances (Golby et al., 2005; Celone et al., 2006; Diamond et al., 2007), as well as their gray matter loss within the hippocampus (Garrido et al., 2002; Rémy et al., 2005).

Besides MTL, episodic memory is subtended by a wide network, including prefrontal and parietal cortices (Cabeza and Nyberg, 2000; Spaniol et al., 2009). These regions are also affected by the neuropathological processes of $\mathrm{AD}$ and show functional abnormalities during episodic memory tasks. Schwindt and Black (2009) reported dorsal, rostral, and medial frontal hypoactivations in $\mathrm{AD}$ patients, as well as in the superior parietal lobule and the precuneus during encoding and/or retrieval. Hence, $\mathrm{AD}$ is characterized more by a functional alteration of episodic memory networks than an isolated deficit in a particular region (Schröder et al., 2001).

Alongside these different functional disturbances, AD patients also show hyperactivations relative to healthy subjects during episodic memory tasks, mainly in fronto-temporal and fronto-parietal regions during encoding and retrieval respectively (Schwindt and Black, 2009). These hyperactivations have been interpreted ever since the earliest studies (Becker et al., 1996; Bäckman et al., 1999) as reflecting compensatory mechanisms, implemented to cope with the neuropathological process. This hypothesis is supported by positive correlations found between episodic memory performances and prefrontal activity during episodic memory tasks (Rémy et al., 2005; Diamond et al., 2007) and negative correlations between hippocampal atrophy and prefrontal activity during retrieval (Garrido et al., 2002; Rémy et al., 2005). Thus, an increasing prefrontal activity may partially compensate hippocampal atrophy and be beneficial for episodic memory functioning.

Functional imaging during episodic memory tasks has also enabled to reveal a deactivation dysfunction in AD. The concept of deactivation has been introduced to give an account of the higher activity in some brain regions during rest, or passive task conditions, than during constrained cognitive activity (Mevel et al., 2011). These brain areas appear to be more engaged during rest and to deactivate during task performance. These deactivations probably allow reallocation of processing resources toward cerebral regions involved in the task (Rombouts et al., 2005). They consistently occur within a network of functionally connected regions, named the default mode network (DMN), which includes prefrontal dorsal and ventral medial regions, posteromedial cortex (posterior cingulate, precuneus, and retrosplenial cortex) and inferior parietal areas. Compared to controls, AD patients show less deactivation in this network, especially in medial parietal areas, during episodic memory processes (Celone et al., 2006; Petrella et al., 2007; Pihlajamäki et al., 2010). As MTL hypoactivations, DMN dysfunction may give an account of AD episodic memory performance deficits (Celone et al., 2006; Petrella et al., 2007).

Thus, despite some evidence of compensatory mechanisms in $\mathrm{AD}$, it remains unclear whether the observed hyperactivations are genuine or reflect this deactivation disturbance. Indeed, lower deactivation in DMN regions could induce patients' hyperactivation in subtraction analyses, as less deactivation represents more activation. However, it is unlikely that this functional alteration would reflect compensatory mechanisms as DMN deactivations play an important role in efficient memory processes (Daselaar et al., 2004). To clarify this issue and to identify the cerebral substrates of memory impairment in $\mathrm{AD}$, we conducted a positron emission tomography (PET) study in $\mathrm{AD}$ patients and elderly subjects, during both encoding and cued-recall in episodic memory, which has seldom been performed in AD. Our study also included control conditions with a semantic demand, to focus our analysis on episodic memory processes, as well as four resting conditions to obtain accurate resting cerebral blood flow. By using rest as a reference, we wished to evaluate the degree of activations during target and control conditions in hyperactivated regions in $\mathrm{AD}$ and determine if they represent a real increase in activity.

\section{MATERIALS AND METHODS}

Eleven newly diagnosed patients with probable $\mathrm{AD}$ (seven women and four men; mean age $77.4 \pm 3.4$ years) were recruited at the University Hospital of Caen. All patients underwent standard neurological and neuropsychological examinations. Patients were selected according to NINCDS-ADRDA criteria (McKhann et al., 1984), attesting of probable AD. They did not present other neurologic pathology or psychiatric antecedent. In order to ensure they could achieve the memory task under the tomograph, a minimum score of 19 at the MMSE (Folstein et al., 1975) was required. This restricted the inclusion to patients at a relatively early stage of the disease. Patients MMSE mean was $24.3( \pm 3.3)$. At the time of the experiment, no patient was taking any medication likely to influence the cognitive state or cerebral blood flow and all were right handed (Edinburgh Handedness Inventory) and French native speakers.

Twelve healthy elderly subjects (six women and six men; mean age $59.1 \pm 2.5$ years) also participated in our study. They were significantly younger than the patients $(p<0.001)$. They were screened to rule out the presence of medical, psychiatric, or neurological disorders. All were unmedicated, had no memory complaint and had a normal T1- and T2-weighted magnetic resonance imaging (apart from changes expected with normal aging). In order not to select elderly subjects with incipient dementia, only those with high scores on the Mattis Dementia Rating Scale (MADRS; Mattis, 1976) were included ( mean = 141.25 \pm 2.9 ).

All subjects were informed of experimental general modalities and gave their written consent before participating. The research program was approved by the Regional Ethic Committee and the study was in accordance with Helsinki declaration.

\section{EXPERIMENTAL DESIGN}

The experimental design has already been used in young (Bernard et al., 2001) and healthy elderly subjects (Bernard et al., 2007). The control group of the present study includes the same subjects as in Bernard et al. (2007), but image preprocessing and analyses differ. Our design is composed of 12 consecutive scans (injections of $\mathrm{H}_{2} \mathrm{O}^{15}$ ) sustained by each participant during a single PET session. Each scanning session comprises five different conditions, each replicated twice (except for the resting condition which is 
repeated four times). Each condition lasts $2 \mathrm{~min}$ and the whole experiment lasts $2 \mathrm{~h}$ and $30 \mathrm{~min}$.

\section{Encoding}

To pinpoint brain areas supporting intentional encoding, two distinct tasks were contrasted: a reading task (baseline) in which 24 different words were read silently and an intentional encoding task (target) in which subjects were explicitly instructed to read silently and memorize 24 words. In order to prevent covert memorizing during the reading task, this condition was deliberately placed at the beginning of the scanning session. In addition, subjects were unaware of the aim of the study (memory experiment), which was presented as a vocabulary one. Acknowledging the risk of order effects, this choice was dictated by the constraints of our paradigm and represents the best possible compromise in attempting to control cognitive strategies. Finally, to further prevent implicit memorizing, subjects were instructed to count backward for $60 \mathrm{~s}$ after each scan involving the reading condition, by 3 for healthy elderly subjects and by 1 for patients. Prior to the intentional encoding task, subjects were informed that they had to memorize the words. During both conditions, items were sequentially presented in lower case, for $4 \mathrm{~s}$ each, on a computer screen. One second separated the presentation of two stimuli. The four lists of words used in the intentional encoding and reading conditions were matched for word frequency and word length (between 4 and 10 letters), and were counterbalanced across subject groups. To cancel out brain regions involved in semantic processing in the subtraction analysis, subjects were instructed in both conditions to make a living/non-living judgment regarding each word, by pressing on one of two possible buttons of a response pad. Half of the words presented referred to living objects.

To reinforce encoding, a second intentional encoding (out of camera - not recorded) was added only for patients. Placed before cued-recall, it enabled them to optimize their chances to subsequently retrieve information.

\section{Retrieval}

To identify the cerebral areas supporting episodic memory retrieval, two conditions were contrasted: a stem-cued recall task (target) and a stem-completion task (baseline). In the stem-cued recall task, subjects were instructed to recall aloud the words studied during the intentional encoding task. They were shown the two first letters of the words (bigrams), presented in a random order. The stem-completion task consisted in completing bigrams, different from those used during the stem-cued recall task, with the first word coming to mind and beginning with the two letters shown. During each task, 24 bigrams (in lower case) were presented sequentially on a computer screen for $4 \mathrm{~s}$ each, separated by a 1-s interstimulus interval.

All stimuli were displayed in white against a black background, on a monitor placed behind the tomograph. The stimuli were shown to the subject using a mirror positioned above the head.

\section{Rest}

During the four resting scan sessions, subjects were instructed to relax, keep their eyes closed, and not focus their mind on any precise thought.

\section{Scanning sequence}

Due to the constraints imposed by the reading condition, the five conditions were presented in fixed order: rest - reading 1 - reading 2 - intentional encoding 1 - (second intentional encoding 1 for $\mathrm{AD}$ patients) - stem-cued recall 1 - stem-completion 1 - rest intentional encoding 2 - (second intentional encoding 2 for AD patients) - stem-cued recall 2 - stem-completion 2 - rest - rest.

\section{DATA ACQUISITION}

Subjects were scanned while lying supine in a dimly lit and quiet room. A black tent was hung around the PET scanner to ensure total darkness. The head was gently immobilized in a dedicated head-rest. Head position was aligned transaxially to the orbitomeatal line with a laser beam. Measurements of regional distribution of radioactivity were performed with an Siemens ECAT $\mathrm{HR}+\mathrm{PET}$ device with full $3 \mathrm{D}$ volume acquisition allowing the reconstruction of 63 planes (thickness $2.4 \mathrm{~mm}$; axial field-of-view $158 \mathrm{~mm}$; effective resolution is $\sim 4.2 \mathrm{~mm}$ in all directions). Transmission scans were obtained with a ${ }^{68} \mathrm{Ge}$ source prior to emission scans. The duration of each scan was $90 \mathrm{~s}$. About $7 \mathrm{mCi} \mathrm{H}_{2} \mathrm{O}^{15}$ were administered as a slow bolus in the left antecubital vein by means of an automated infusion pump. Each experimental condition started $30 \mathrm{~s}$ before data acquisition and continued until scan completion. This process was repeated for each of the 12 scans, for a total injected dose of $\sim 80 \mathrm{mCi}$. The interval between injections was $7 \mathrm{~min} 40 \mathrm{~s}$. The position of the head was controlled with the laser beam prior to each injection.

\section{DATA ANALYSIS}

Imaging data were pre-processed and analyzed using the SPM5 software (statistical parametric mapping, http://www.fil.ion.ucl.ac. $\mathrm{uk} / \mathrm{spm}$ ). Each reconstructed PET image was realigned and spatially normalized using the MNI $\left[{ }^{15} \mathrm{O}\right]$ PET template. To account for within- and between-subject variations in global cerebral blood flow, the value of each voxel was scaled by the mean gray matter value of the corresponding image, extracted using a mask from our database. A Gaussian filter with a 12-mm FWHM was then applied to smooth each image to compensate for intersubject differences and increase the signal-to-noise ratio. $T$-statistic maps were generated ( $p<0.005$ uncorrected, $k=50$ voxels) in a flexible factorial design allowing to model subject effect. We kept only the clusters also present at a more stringent threshold $(p<0.001$ uncorrected). First, to explore cerebral regions associated with episodic memory encoding, we subtracted reading from intentional encoding maps [corresponding to the contrast (intentional encoding - reading)]. This contrast enabled us to dismiss cerebral structures involved in reading and semantic processes. Second, to study brains areas associated with episodic memory retrieval, we subtracted stem-completion from stem-cued recall imaging maps [corresponding to the contrast (stem-cued recall - stemcompletion)]. This allowed us to remove cerebral regions involved in oral production in order to isolate those associated with item retrieval in episodic memory. These contrasts were conducted for both within- and between- group analyses. Moreover, a conjunction analysis was performed on both contrasts [(intentional encoding - reading) and (stem-cued recall - stem-completion)] to identify brain areas commonly activated in both $\mathrm{AD}$ and controls. 
An inclusive mask was used ( $p<0.05$ uncorrected) to ensure that between group activation differences were effectively located in regions activated by the group for the given contrast. For instance, we masked the contrast [(intentional encoding ${ }_{\mathrm{NC}}$ - reading $\mathrm{NC}_{\mathrm{NC}}$ (intentional encoding $\mathrm{AD}$ - reading $\mathrm{AD}$ )] with the (intentional encoding $\mathrm{NC}-$ reading $\mathrm{NC}$ ) image to restrict the results to brain areas activated by healthy elders during information encoding.

To further investigate the nature of the activation differences between patients and healthy subjects, we performed region of interest (ROI) analyses focused on cerebral regions showing significant between group differences. We extracted the mean signal for each region in each individual pre-processed image. A mean value per ROI was obtained by extraction from individual nonsmoothed images. All values from a same condition were then averaged, combining the values of the two acquisitions for the target conditions and the four acquisitions of the resting condition.

Given that the aim of this study was to investigate the nature of the hyperactivations in patients, the activation profiles of these regions were assessed in both patients and controls. More precisely, we compared the activation intensity during rest to the ones during target (intentional encoding and stem-cued recall) and control (reading and stem-completion) conditions. We used the non-parametrical test of Wilcoxon to perform intra-group comparisons and the non-parametrical test of Mann-Whitney for inter-group comparisons. For all ROI-based analyses, the Statistica software was used and results were considered as significant when $p<0.05$.

\section{RESULTS}

\section{BEHAVIORAL RESULTS}

For the stem-cued recall task under the tomograph, patients and controls retrieved on average $11( \pm 8.14)$ and 20.25 words $( \pm 6.08)$, respectively. The difference between the two groups was significant (Mann-Whitney test; $U=24 ; p<0.01$ ).

\section{IMAGING RESULTS}

\section{Intentional encoding versus reading}

Intra- and inter-group results for intentional encoding minus reading are depicted on the Table $\mathbf{1}$.

Intra-group results. During encoding, $\mathrm{AD}$ patients activated the left middle frontal gyrus, medial frontal gyrus, and anterior cingulate cortex compared to reading. Some temporal regions (right middle and transverse gyri) and parietal (right inferior lobule and posterior cingulate cortex) were also more activated during intentional encoding than reading. Unlike patients, healthy elderly subjects showed activations in the right parahippocampal gyrus, extending to the hippocampus. Other activations were found in left frontal (precentral, middle and superior gyri, and anterior cingulate cortex) and parietal (superior parietal lobule and precuneus) areas.

Inter-group results. Inter-group analyses highlighted hypoactivations, in $\mathrm{AD}$ patients, in the right posterior parahippocampal and hippocampus (see Figure 1A), as well as in the left anterior cingulate cortex. Compared to the control group, $\mathrm{AD}$ patients showed significantly higher activation in the medial frontal gyrus, anterior cingulate cortex, and inferior parietal lobule of the right hemisphere (see Figure 2A). The conjunction analysis did not show any regions of common activation between the two groups.

ROI analyses results. The results of the ROI analyses, focused on regions showing more activation in $\mathrm{AD}$ than healthy subjects, are presented on Figure 2A. Healthy elderly subjects showed significantly more activation during rest than encoding in the anterior part of the cingulate cortex $(T=13, Z=2.04, p<0.05)$ and medial frontal cortex $(T=7, Z=2.51, p<0.05)$. In other words, both regions were significantly deactivated during encoding in the control group. In contrast, $\mathrm{AD}$ patients solely deactivated the medial frontal region ( $T=8, Z=2.22, p<0.05)$. Inter-group comparison revealed a significant difference in the anterior cingulate cortex $(U=32, p<0.05)$, indicating that the magnitude of deactivation was higher in control subjects than in patients. Concerning the comparisons between control (reading) and rest conditions, differences were only observed in the patient group: the medial frontal cortex $(T=0, Z=2.93, p<0.005)$ and inferior parietal lobule $(T=2, Z=2.76, p<0.01)$ showed significantly more activation during rest than reading. The analyses also highlighted a significant group effect with higher deactivation in patients than in controls during the reading task in both regions (medial frontal cortex: $U=21, p<0.005$; inferior parietal lobule: $U=15, p<0.001)$.

\section{Stem-cued recall versus stem-completion}

Intra- and inter-group results for stem-cued recall minus stemcompletion are depicted on the Table 2 .

Intra-group results. Patients showed activations mainly located in the right frontal lobe (middle and superior gyri) and parietal regions (right supramarginal gyrus and bilateral posterior cingulate cortex) when stem-completion activations were subtracted to stem-cued recall activations. Healthy elderly subjects showed significant activations in the bilateral parahippocampal region (uncus, hippocampus, and amygdala) and a broad right prefrontal area (inferior, middle, superior gyri, and medial frontal gyrus). In addition, control subjects also activated the right anterior insula, bilateral parietal cortex (left supramarginal gyrus and bilateral inferior parietal lobe), right cuneus, and left cerebellum.

Inter-group results. Alzheimer's disease patients presented less activation than controls in the left parahippocampal gyrus (see Figure 1B). Patients' hypoactivations were also observed in the middle and superior right frontal gyri, and in the left parietal lobe (inferior parietal lobule extending into the supramarginal and superior temporal gyri), the bilateral occipital cortex and cerebellum. In contrast, patients presented hyperactivations, compared to the control group, in the right middle cingulate cortex encompassing paracentral and precentral gyri, and in the bilateral precuneus (see Figure 2B). Finally, a cluster in the right middle and superior frontal gyrus was observed in the conjunction analysis.

ROI analyses results. The results of the ROI analyses are presented on Figure 2B. The mean signal analyses within AD hyperactivated regions indicated that the right middle cingulate cluster $(T=0, Z=3.06, p<0.005)$, as well as both clusters 
Table 1 | Significant activations for intra- and inter-group comparisons for the (encoding - reading) contrast ( $p<0.005$ uncorrected, cluster extent $>50$ voxels).

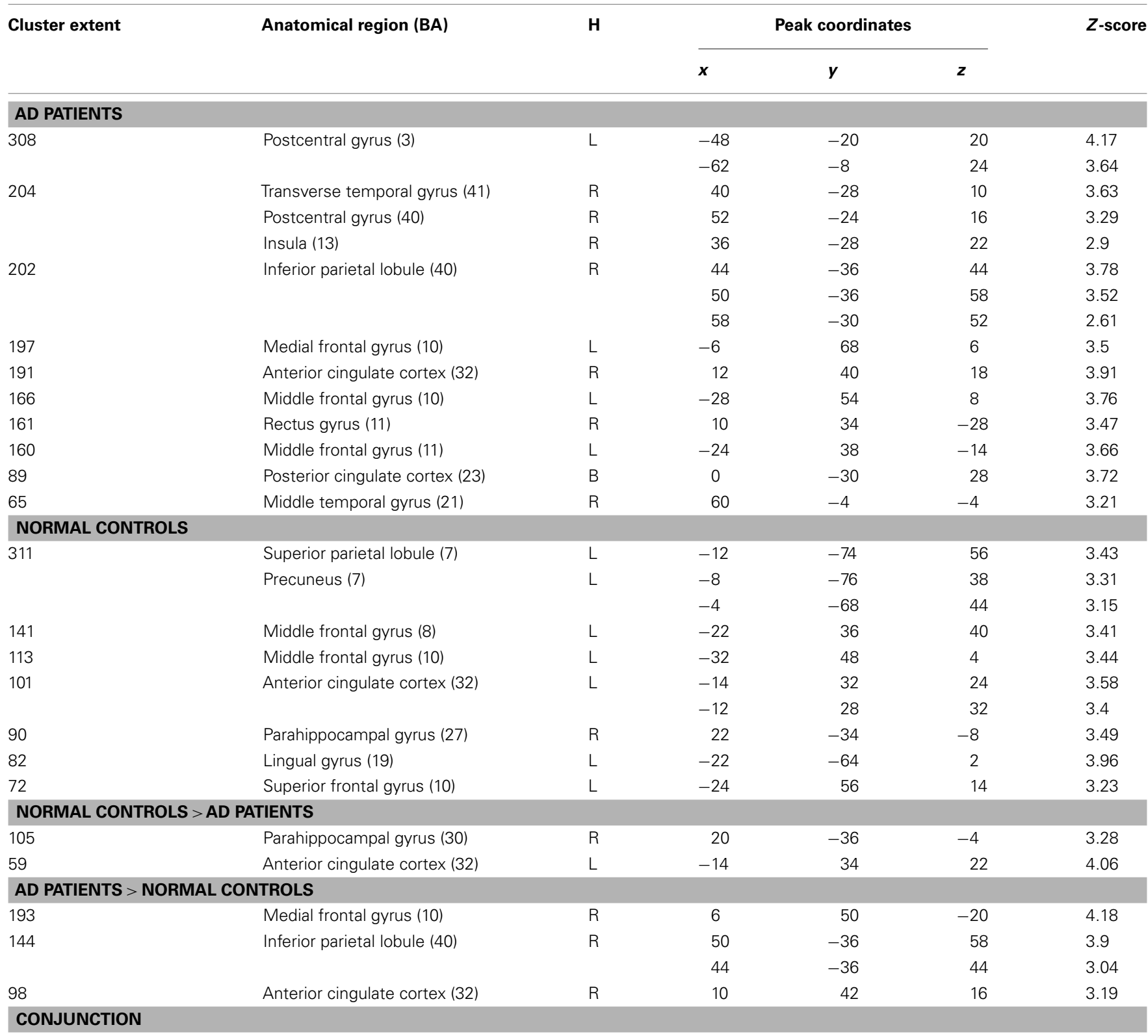

No significant cluster

Coordinates are in MNI space. BA, Brodmann area; $H$, hemisphere; $B$, bilateral; $L$, left; $R$, right.

in the precuneus $(T=5, Z=2.67, p<0.01 ; T=6, Z=2.59$, $p<0.01$ ) showed more activation during rest than during the cued recall condition in the healthy group. Within the $\mathrm{AD}$ patient group, none of these regions presented significant activation differences between the resting and target conditions. Inter-group analyses highlighted a significant higher difference between resting and cued recall conditions in controls, compared to patients, in all the hyperactivated regions (middle cingulate cluster: $U=13, p<0.001$; both precuneus clusters: $U=30, p<0.05$ ). With respect to the stem-completion and rest comparisons, differences were only observed in the patient group: the right middle cingulate $(T=3, Z=2.67, p<0.01)$ and left precuneus $(T=7$, $Z=2.31, p<0.05)$ showed a significantly higher mean signal during rest than in the control condition. Inter-group analyses revealed a significant difference in the right middle cingulate $(U=27, p<0.05)$, with higher deactivation in $\mathrm{AD}$ patients than in healthy subjects.

\section{DISCUSSION}

The main objective of this study was to improve our current knowledge concerning the cerebral substrates of episodic memory disorder in $\mathrm{AD}$ and further explore the hyperactivation 

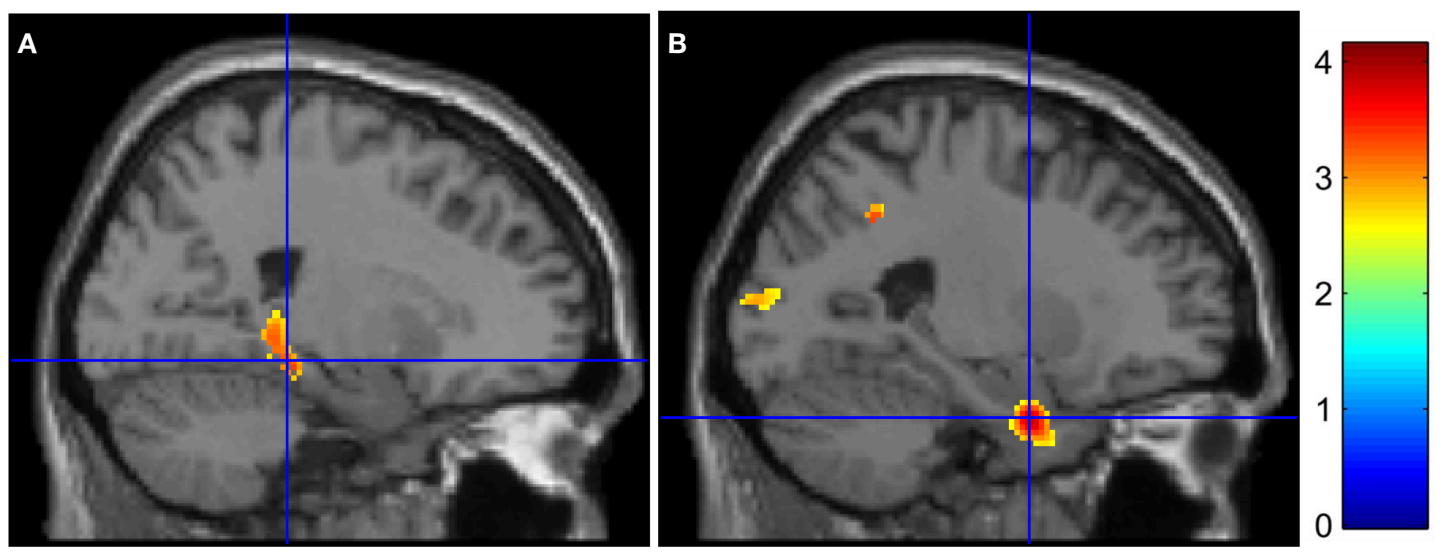

FIGURE 1 | Medial temporal lobe hypoactivations in AD patients for (encoding - reading) and (stem-cued recall - stem-completion) contrasts (respectively A and B). The color bar indicates $T$-values.

phenomenon, frequently reported in the functional imaging literature. To this end, we compared the cerebral blood flow of $\mathrm{AD}$ patients at a relatively early stage of the disease to healthy controls during both encoding and retrieval in episodic memory. Subtraction analyses were performed to highlight regions showing functional alteration in AD. Further analyses were then conducted within regions showing hyperactivation in $\mathrm{AD}$ to investigate the nature of these cerebral modifications. To this end, activity was compared within each group during target and control conditions versus rest.

\section{FUNCTIONAL DISORDERS WITHIN THE NETWORK SUSTAINING EPISODIC MEMORY PROCESSES}

Our results indicate a functional dysfunction in AD patients in several brain regions during information encoding and retrieval in episodic memory. Whatever the process involved, patients did not present any MTL activation and this region was hypoactivated relative to controls. This result is congruent with AD functional imaging literature that consistently reported MTL hypoactivation during encoding (Machulda et al., 2003; Sperling et al., 2003; Dickerson et al., 2005; Golby et al., 2005; Pariente et al., 2005; Rémy et al., 2005; Celone et al., 2006; Petrella et al., 2007) and retrieval (Bäckman et al., 1999; Garrido et al., 2002; Grön et al., 2002; Pariente et al., 2005; Rémy et al., 2005). This functional perturbation during retrieval has been previously related to hippocampal atrophy (Garrido et al., 2002; Rémy et al., 2005). The presence of neuropathological abnormalities within this cerebral structure (Braak and Braak, 1991), as well as the disconnexion with other brain areas (Delbeuck et al., 2003; Villain et al., 2008), may also explain this perturbation. Given its core role in episodic memory (Viard et al., 2007), the dysfunction of the MTL is coherent with the deficit observed in our AD patients.

In addition to MTL hypoactivation, AD patients showed lower activation within the left anterior cingulate cortex during encoding compared to control subjects. A similar dysfunction during encoding has been observed in several functional imaging studies concerning AD (Stern et al., 2000; Rombouts et al., 2005; Celone et al., 2006; Petrella et al., 2007). Using an associative learning paradigm, Petrella et al. (2007) noticed that AD patients showed significantly lower activation in this region compared to patients with a mild cognitive impairment (MCI), who themselves showed lower activation than healthy elderly subjects. Moreover, activity in the medial frontal region was found to correlate with performances in healthy elderly, AD and MCI patients (Celone et al., 2006). The authors showed that the medial frontal and anterior cingulate cortices are part of a broader network, which does not present task-related activity, but maintains its activity throughout the experiment. This result, as well as the role conferred to the anterior cingulate region in the attentional system (Cabeza and Nyberg, 2000), suggests that hypoactivation in AD may reflect a dysfunction in the modulation of attentional resources during encoding.

During stem-cued recall, apart from the left MTL (see above), inter-group analyses mainly showed hypoactivations in the right middle and superior frontal gyri. Activation in this region was not only less important but was also less extended in $\mathrm{AD}$ patients. $A$ visual inspection of intra-group results revealed that most $A D$ patients' frontal activations overlapped with regions activated by controls (highlighted by the conjunction analysis), whereas the reverse was not true. Hypoactivation in this brain area, and notably in the anterior prefrontal gyrus (Brodmann area, BA 10), was also mentioned in the meta-analysis of functional imaging studies in episodic memory in AD (Schwindt and Black, 2009). This cerebral structure has a role in the "retrieval mode," a neurocognitive state in which subjects maintain an attentional focus on a particular past episode during retrieval (Lepage et al., 2000). According to this hypothesis, regions involved in retrieval mode, also known as REMO sites (REtrieval MOde), are activated regardless of the efficiency of retrieval. Considering our results, the decreasing recruitment of the right anterior prefrontal gyrus may denote a deficit in $\mathrm{AD}$ patients to reach this retrieval neurocognitive state and may consequently contribute to their poor performance.

The decreased activation in the inferior parietal gyrus (BA 40) observed during the stem-cued recall task has also been reported in $\mathrm{AD}$ during retrieval, in the left (Bäckman et al., 1999; Garrido et al., 2002) and right (Rémy et al., 2005) hemispheres. This region 


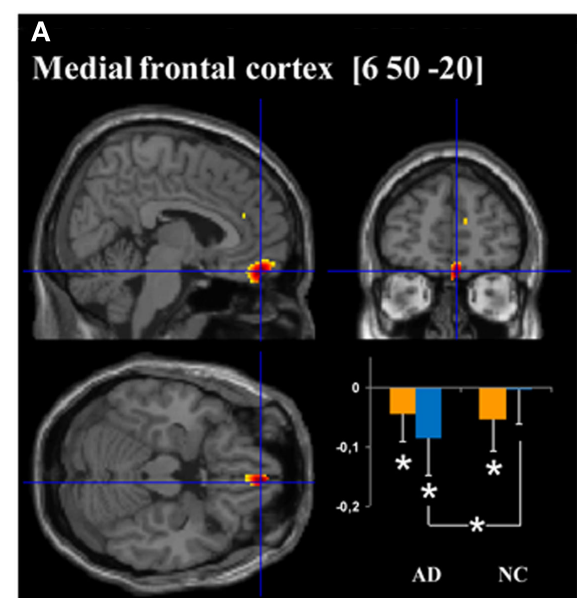

Inferior parietal lobule [ [50 -36 58]
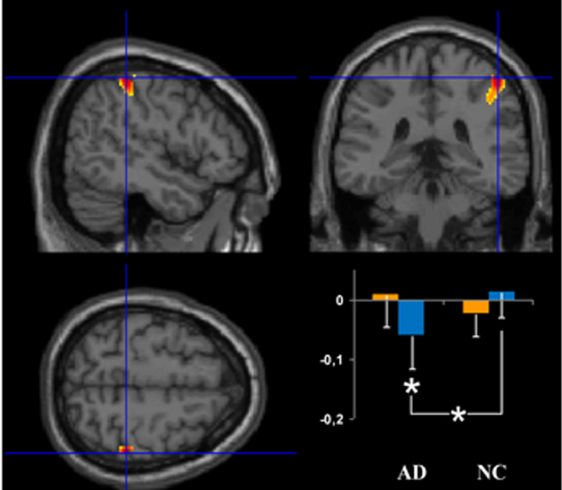

Anterior cingulate cortex [10 42 16]

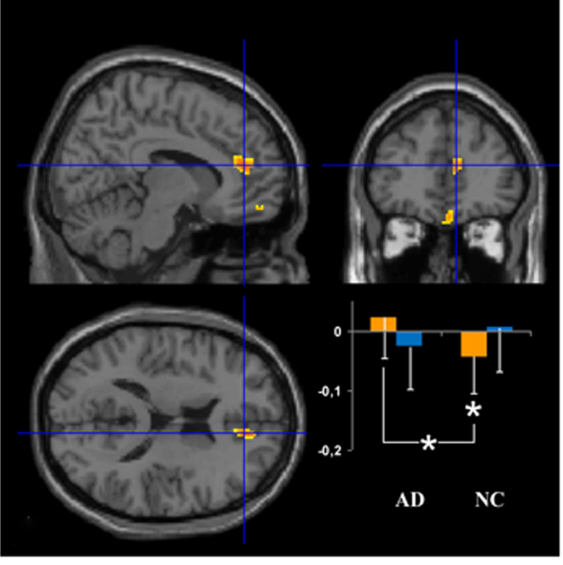

FIGURE 2 | Cerebral regions showing hyperactivation in AD patients compared to normal controls (NC) for

(encoding - reading) and (stem-cued recall - stem-completion) contrasts (respectively A and B). Plots represent the signal change during target (orange bars) and control (blue bars) conditions relative to rest in $\mathrm{AD}$ and $\mathrm{NC}$ groups. More precisely, the orange bars correspond

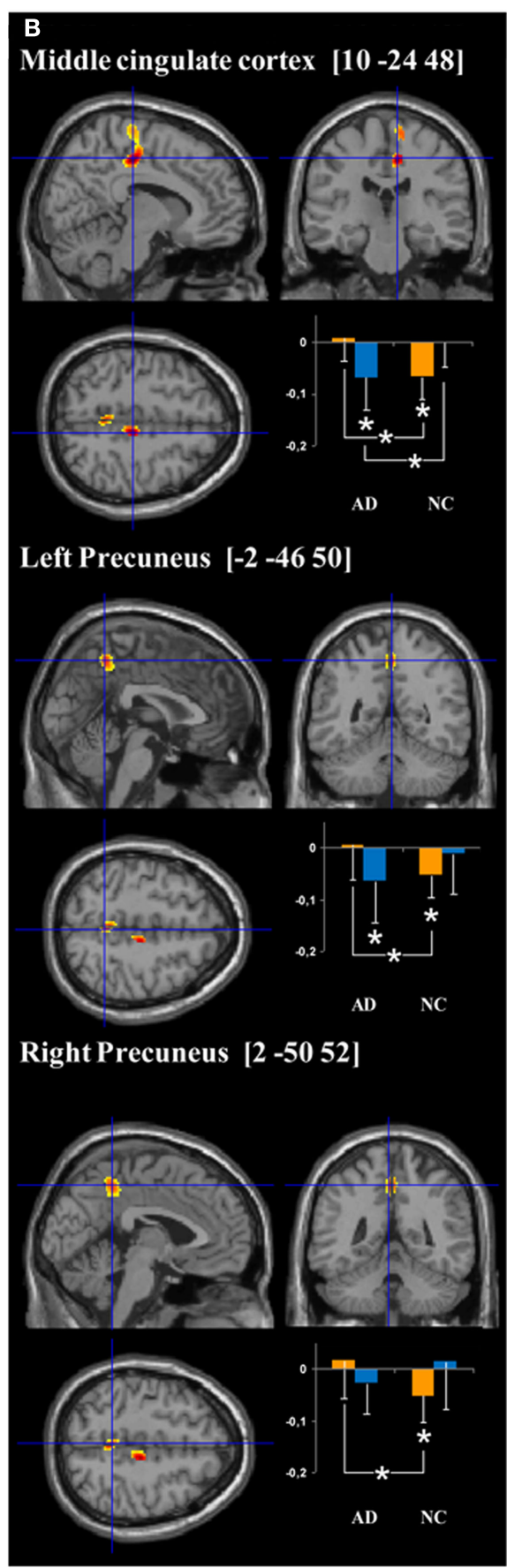

to the mean intensity of the subtraction target - rest conditions (i.e., "intentional encoding - rest" in A and "stem-cued recall - rest" in B) and the blue bars to the mean intensity of the subtraction control - rest conditions (i.e., "reading - rest" in $\mathbf{A}$ and "stem-completion - rest" in the B). The units correspond to the mean intensity of the scaled CBF values in the ROIs. ${ }^{*} p<0.05$. is among the first parietal region to present an atrophy and a metabolic dysfunction in $\mathrm{AD}$ (for review, see Jacobs et al., 2012), this latter being more important than the gray matter loss (Chételat et al., 2008). According to the Attention to Memory hypothesis (AtoM; Ciaramelli et al., 2008) developed in order to explain parietal activations during recall, inferior parietal structures underlie 
Table 2 | Significant activations for intra- and inter-group comparisons for the (stem-cued recall - stem-completion) contrast ( $p<0.005$ uncorrected, cluster extent $>50$ voxels).

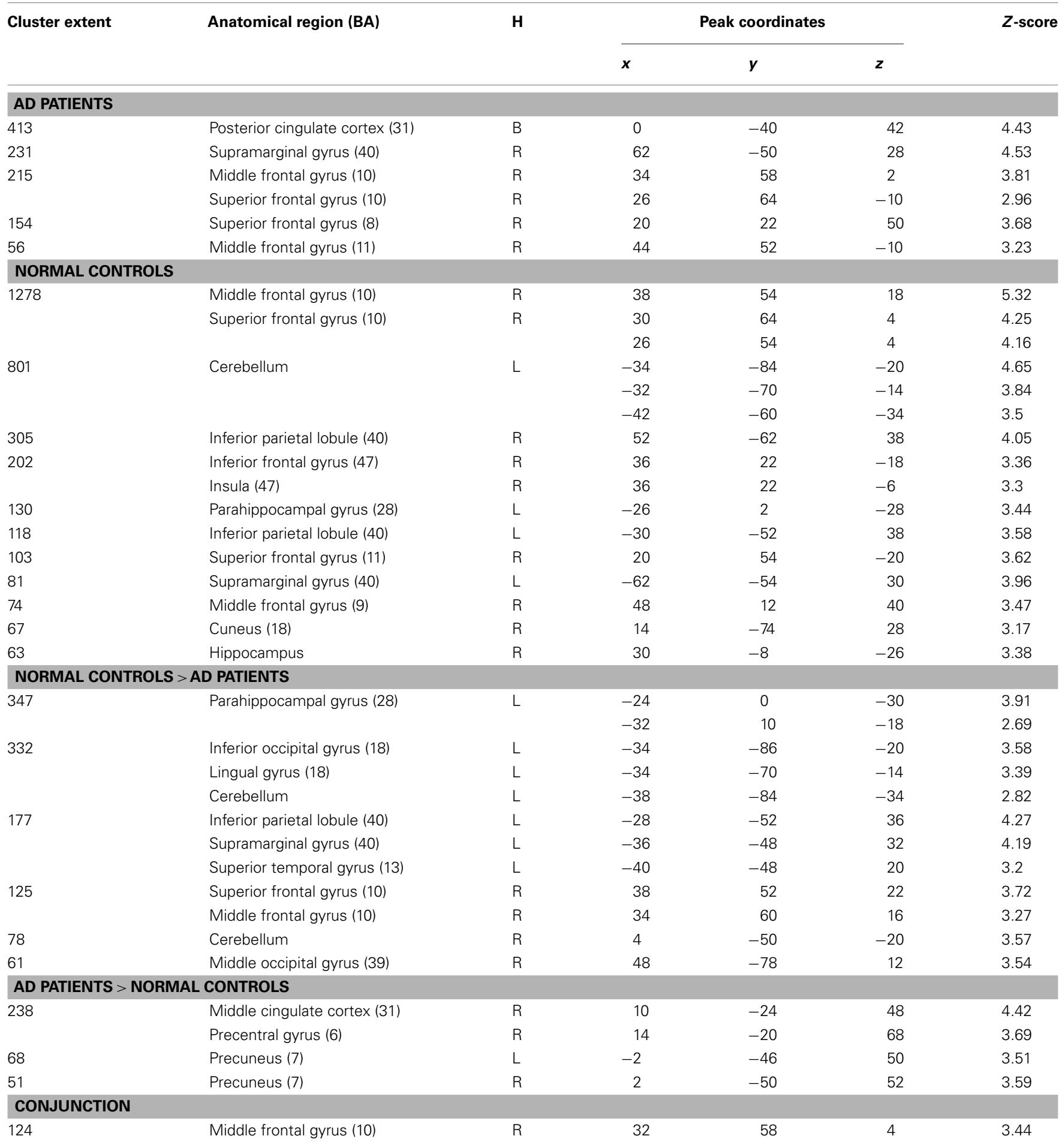

Coordinates are in MNI space. BA, Brodmann area; $H$, hemisphere; $B$, bilateral; $L$, left; $R$, right.

attentional processes during direct retrieval in episodic memory. More precisely, these brain areas may "mediate the automatic attentional capture by the recollected memory, which might be necessary for the memory to enter consciousness, and therefore be experienced as a memory" (Ciaramelli et al., 2008). According to the authors, its activity is all the more important since 
memories are strong and accompanied by vivid remembering. Our AD patients' inability to recruit this region like controls may consequently compromise the conscious access to their memories and an effective retrieval in episodic memory.

\section{HYPERACTIVATION NATURE AND DEACTIVATION IMPAIRMENT IN THE DEFAULT MODE NETWORK}

One of the main aims of this study was to clarify the nature of AD patients' hyperactivations observed during episodic memory tasks. Inter-group analysis showed hyperactivation in the right inferior parietal lobule and anterior medial frontal region during encoding. Some previous studies already found hyperactivation in AD in these regions (Gould et al., 2005; Pariente et al., 2005). Concerning the cued-recall condition, patients showed more activation than controls in the bilateral precuneus, middle cingulate cortex, and precentral gyrus. Again, some of these regions have been previously reported as hyperactivated in $\mathrm{AD}$ in episodic (Gould et al., 2005; Schwindt and Black, 2009) or autobiographical (Meulenbroek et al., 2010) memory studies.

To understand the nature of these hyperactivations, we extracted the mean signal within each of these regions and compared their activity during target (encoding or cued-recall), control (reading or stem-completion) and resting conditions. The first comparison (between target and rest conditions) indicated that all of these regions (except for the inferior parietal lobule) were significantly more active during rest than during the target conditions in healthy controls. In other words, these areas deactivated during episodic memory encoding and retrieval. In $\mathrm{AD}$ patients, only the anterior medial frontal region presented a significant deactivation during encoding. Furthermore, the anterior cingulate cortex during encoding, as well as the middle cingulate cortex and precuneus during retrieval, were significantly more deactivated in controls than in patients. Hence, our results indicate that none of the hyperactivated regions in our $\mathrm{AD}$ group were significantly activated relative to rest either in patients or in the healthy group. The fact that they appeared to be hyperactivated during encoding and retrieval in episodic memory results more from a deficit in deactivation during the target conditions than a real increased activity in AD.

Deactivation disturbance in some of these regions, especially in the medial parietal regions during recall, is coherent with the AD literature. Indeed, Petrella et al. (2007) reported a continuum in the deactivation magnitude in the precuneus and posterior cingulate cortex during encoding. AD patients showed a deactivation of lower intensity than MCI patients, who themselves deactivated less intensively than healthy controls. Deactivation dysfunction within these regions has been reported in $\mathrm{AD}$ during encoding memory tasks (Celone et al., 2006), but also during semantic (Lustig et al., 2003) and working (Rombouts et al., 2005) memory tasks. A similar disturbance has also been observed in individuals with genetic risk for $\mathrm{AD}$ during incidental encoding (Persson et al., 2008). The alteration of deactivation in these regions, important for efficient memory processes (Daselaar et al., 2004), appears prejudicial to AD patients' memory performances (Celone et al., 2006; Petrella et al., 2007). It may ensue from the massive presence of amyloid plaques in these regions (Sperling et al., 2010) or result from MTL connection disruption, as magnitude of deactivation has been related to MTL activation in AD (Celone et al., 2006; Pihlajamäki et al., 2008). To our knowledge, our study provides the first evidence, although indirect, of a deactivation dysfunction in $\mathrm{AD}$ during episodic memory retrieval.

Regarding the anterior cingulate cortex, the deficit of deactivation of this structure during an episodic memory task would be more dependent on an aging effect than on the pathology (Lustig et al., 2003; Gould et al., 2006). Aging is accompanied by brain functional modifications, including reduction in the activity measured at rest and the magnitude of deactivations during task performance (for a review on $\mathrm{DMN}$ in aging and $\mathrm{AD}$, see Mevel et al., 2011). However, it should be noted that abnormalities in the temporal pattern of deactivation in the anterior cingulate cortex have already been observed in $\mathrm{AD}$ during encoding (Rombouts et al., 2005) and disease effect should not be excluded.

Finally, our ROI analyses also emphasized group differences during the control conditions (reading and stem-completion) relative to rest. The medial frontal cortex and the inferior parietal lobule during reading, as well as the right middle cingulate and left precuneus during stem-completion, showed a significant deactivation in patients but not in controls. Additionally, among these four regions, only the left precuneus did not show a higher deactivation during the control conditions in patients compared to controls. These results reveal excessive deactivation in these regions during the control tasks in $\mathrm{AD}$ patients which also accounts for their hyperactivations observed in the subtraction analyses.

Note that we performed additional analyses (Spearman correlation) to explore the relations between the mean signal in the ROIs and performances (data not shown). These correlations were performed separately in each group. Among patients, no significant correlation was observed between performances and the amplitude of activation during target conditions relative to either the baseline (rest) or the control conditions. This result highlights that patients' hyperactivations, in addition to not representing increased activations, are not sustaining their performances. Interestingly, one significant negative correlation was obtained in controls $(r=-0.63, p<0.05)$ in the right medial frontal gyrus suggesting that higher deactivation during encoding (relative to the reading condition) was associated with better performances, a result which reinforces our interpretation.

Overall, our results attest of the interest to use resting-state activity as a reference condition in order to better understand activation alterations in $\mathrm{AD}$. Disease functional modifications during control and target conditions may lead to hyperactivations when they are subtracted. Nevertheless, these hyperactivations may not systematically represent increased activity and precautions should be taken when interpreting such results in terms of compensatory mechanisms.

\section{LIMITATIONS}

We must mention some limitations of the present study, leading us to moderate our results. First, patients were significantly older than healthy control subjects. Aging is known to be accompanied by functional modifications during episodic memory task and hypoactivation, hyperactivation and deactivation decreases have been reported in healthy elderly subjects (for review about 
encoding alteration in aging and AD, see Sperling, 2007). In this respect, some of our results may attest of an aging effect rather than $\mathrm{AD}$ per se, as pointed out for the anterior cingulate cortex deactivation impairment. Nevertheless, we found coherent results with the current $\mathrm{AD}$ literature and hence believe they mainly reflect the disease effects. Second, we were unable to perform optimal spatial normalization given the absence of anatomical MRIs in some subjects. This methodological bias may lead to an inaccurate spatial location. We were also not able to correct for partial volume effect, inherent to the low spatial resolution of PET which induces a contamination of cerebral blood flow values by neighboring voxels. This effect, particularly sensitive to small brain regions, could be exacerbated by atrophic processes present in $\mathrm{AD}$ (Mevel et al., 2007). Yet, our results were located in cerebral regions congruent with previous studies and hence were unlikely to represent noise. Finally, it is important to note that some of our subtraction analyses results did not survive a cluster-level threshold of $p<0.05$ and part of our ROI results failed to reach significance after a Bonferroni correction for the number of comparisons. This lack of statistical power is likely due to our small sample sizes, as well as to the properties of PET itself (relatively noisy image, low spatial resolution...). Future studies must be conducted to replicate our results and should control for variables likely to influence functional activity, such as cognitive reserve or task difficulty (Gould et al., 2005, 2006; Solé-Padullés et al., 2009).

\section{REFERENCES}

Bäckman, L., Andersson, J. L., Nyberg, L., Winblad, B., Nordberg, A., and Almkvist, O. (1999). Brain regions associated with episodic retrieval in normal aging and Alzheimer's disease. Neurology 52, 1861-1870.

Becker, J. T., Mintun, M. A., Aleva, K., Wiseman, M. B., Nichols, T., and DeKosky, S. T. (1996). Compensatory reallocation of brain resources supporting verbal episodic memory in Alzheimer's disease. Neurology 46, 692-700.

Bernard, F., Desgranges, B., Platel, H., Baron, J. C., and Eustache, F. (2001). Contributions of frontal and medial temporal regions to verbal episodic memory: a PET study. Neuroreport 12, 1737-1741.

Bernard, F. A., Desgranges, B., Eustache, F., and Baron, J.-C. (2007). Neural correlates of age-related verbal episodic memory decline: a PET study with combined subtraction/correlation analysis. Neurobiol. Aging 28, 1568-1576.

Braak, H., and Braak, E. (1991). Neuropathological stageing of Alzheimer-related changes. Acta Neuropathol. 82, 239-259.

Cabeza, R., and Nyberg, L. (2000). Imaging cognition II: an empirical review of 275 PET and fMRI studies. J. Cogn. Neurosci. 12, 1-47.
Celone, K. A., Calhoun, V. D., Dickerson, B. C., Atri, A., Chua, E. F., Miller, S. L., DePeau, K., Rentz, D. M., Selkoe, D. J., Blacker, D., Albert, M. S., and Sperling, R. A. (2006). Alterations in memory networks in mild cognitive impairment and Alzheimer's disease: an independent component analysis. J. Neurosci. 26, 10222-10231.

Chételat, G., Desgranges, B., de La Sayette, V., Viader, F., Berkouk, K., F., Dupuy, B., Hannequin, D., Baron, J. C., and Eustache, F. (2003). Dissociating atrophy and hypometabolism impact on episodic memory in mild cognitive impairment. Brain 126, 1955-1967.

Chételat, G., Desgranges, B., Landeau, B., Mézenge, F., Poline, J. B., de La Sayette, V., Viader, F., Eustache, F., and Baron, J.-C. (2008). Direct voxel-based comparison between grey matter hypometabolism and atrophy in Alzheimer's disease. Brain 131, 60-71.

Ciaramelli, E., Grady, C. L., and Moscovitch, M. (2008). Top-down and bottom-up attention to memory: a the posterior parietal cortex in memory retrieval. Neuropsychologia 46, 1828-1851.

Daselaar, S. M., Prince, S. E., and Cabeza, R. (2004). When less means more: Landeau, B., Lalevée, C., Le Doze, hypothesis (AtoM) on the role of

\section{CONCLUSION}

The aim of this study was to further understand the cerebral structures supporting episodic memory performance in $\mathrm{AD}$ and clarify patients' hyperactivations, frequently interpreted as compensatory mechanisms to cope with the neuropathological process. In accordance with the $\mathrm{AD}$ functional imaging literature, we observed functional disturbance within core episodic memory areas, particularly in MTL structures. Our results also suggest a wider episodic memory network impairment, in terms of both task-related activations and deactivations. Common activations between controls and $\mathrm{AD}$ patients were only found in a right frontal area during retrieval which was broader in healthy controls than in patients. This network disturbance may partially reflect the intricacy of impaired cognitive processes. Notably, the attentional system could contribute to patients' episodic memory deficits, as its cerebral network proved to be disturbed. This study also highlighted that most $\mathrm{AD}$ patients' hyperactivations actually reflected deactivation abnormalities. It remains difficult to evaluate the impact of this message given previous work reported mostly $\mathrm{AD}$ hyperactivations in dorsolateral or ventrolateral prefrontal regions, which was not the case here. Thus, although genuine compensatory mechanisms may exist in $\mathrm{AD}$, our work emphasizes the caution that is necessary to interpret hyperactivations in this way. This kind of interpretation, easily invoked in the literature, would have been specious in our experiment.

deactivations during encoding that predict subsequent memory. $\mathrm{Neu}$ roimage 23, 921-927.

Delbeuck, X., Van der Linden, M. and Collette, F. (2003). Alzheimer's disease as a disconnection syndrome? Neuropsychol. Rev. 13, 79-92.

Desgranges, B., Baron, J. C., de La Sayette, V., Petit-Taboué, M. C., Benali, K., Landeau, B., Lechevalier, B., and Eustache, F. (1998). The neural substrates of memory systems impairment in Alzheimer's disease. A PET study of resting brain glucose utilization. Brain 121, 611-631.

Diamond, E. L., Miller, S., Dickerson, B. C., Atri, A., DePeau, K., Fenstermacher, E., Pihlajamäki, M., Celone, K., Salisbury, S., Gregas, M., Rentz, D., and Sperling, R. A. (2007). Relationship of fMRI activation to clinical trial memory measures in Alzheimer disease. Neurology 69, 1331-1341.

Dickerson, B. C., Salat, D. H., Greve, D. N., Chua, E. F., Rand-Giovannetti, E., Rentz, D. M., Bertram, L., Mullin, K., Tanzi, R. E., Blacker, D., Albert, M. S., and Sperling, R. A. (2005). Increased hippocampal activation in mild cognitive impairment compared to normal aging and AD. Neurology 65, 404-411.
Duyckaerts, C., Delatour, B., and Potier, M.-C. (2009). Classification and basic pathology of Alzheimer disease. Acta Neuropathol. 118, 5-36.

Folstein, M. F., Folstein, S. E., and McHugh, P. R. (1975). "Mini-mental state". A practical method for grading the cognitive state of patients for the clinician. J. Psychiatr. Res. 12, 189-198.

Garrido, G. E. J., Furuie, S. S., Buchpiguel, C. A., Bottino, C. M. C., Almeida, O. P., Cid, C. G., Camargo, C. H. P., Castro, C. C., Glabus, M. F., and Busatto, G. F. (2002). Relation between medial temporal atrophy and functional brain activity during memory processing in Alzheimer's disease: a combined MRI and SPECT study. J. Neurol. Neurosurg. Psychiatr. 73, 508-516.

Golby, A., Silverberg, G., Race, E. Gabrieli, S., O'Shea, J., Knierim, K., Stebbins, G., and Gabrieli, J. (2005). Memory encoding in Alzheimer's disease: an fMRI study of explicit and implicit memory. Brain 128, 773-787.

Gould, R. L., Brown, R. G., Owen, A. M., Bullmore, E. T., and Howard, R. J. (2006). Task-induced deactivations during successful paired associates learning: an effect of age but not Alzheimer's disease. Neuroimage 31, 818-831. 
Gould, R. L., Brown, R. G., Owen, A. M., Bullmore, E. T., Williams, S. C. R., and Howard, R. J. (2005). Functional neuroanatomy of successful paired associate learning in Alzheimer's disease. Am. J. Psychiatry 162, 2049-2060.

Grön, G., Bittner, D., Schmitz, B., Wunderlich, A. P., and Riepe, M. W. (2002). Subjective memory complaints: objective neural markers in patients with Alzheimer's disease and major depressive disorder. Ann. Neurol. 51, 491-498.

Jacobs, H. I. L., Van Boxtel, M. P. J., Jolles, J., Verhey, F. R. J., and Uylings, H. B. M. (2012). Parietal cortex matters in Alzheimer's disease: an overview of structural, functional and metabolic findings. Neurosci. Biobehav. Rev. 36, 297-309.

Lepage, M., Ghaffar, O., Nyberg, L., and Tulving, E. (2000). Prefrontal cortex and episodic memory retrieval mode. Proc. Natl. Acad. Sci. U.S.A. 97, 506-511.

Lustig, C., Snyder, A. Z., Bhakta, M., O’Brien, K. C., McAvoy, M., Raichle, M. E., Morris, J. C., and Buckner, R. L. (2003). Functional deactivations: change with age and dementia of the Alzheimer type. Proc. Natl. Acad. Sci. U.S.A. 100, 14504-14509.

Machulda, M. M., Ward, H. A., Borowski, B., Gunter, J. L., Cha, R. H., O'Brien, P. C., Petersen, R. C., Boeve, B. F., Knopman, D., Tang-Wai, D. F., Ivnik, R. J., Smith, G. E., and Tangalos, E. G. (2003). Comparison of memory fMRI response among normal, MCI, and Alzheimer's patients. Neurology 61, 500-506.

Mattis, S. (1976). "Mental status examination for organic mental syndrome in the elderly patient," in Geriatric Psychiatry: A Handbook for Psychiatrists and Primary Care Physicians, eds L. Bellak and T. B. Karasu (New York: Grune \& Stratton), 77-121.

McKhann, G. M., Drachman, D., Folstein, M., Katzman, R., Price, D., and Stadlan, E. M. (1984). Clinical diagnosis of Alzheimer's disease: report of the NINCDS-ADRDA Work Group under the auspices of Department of Health and Human Services Task Force on Alzheimer's Disease. Neurology 34, 939-944.

McKhann, G. M., Knopman, D. S., Chertkow, H., Hyman, B. T., Jack, C. R. Jr., Kawas, C. H., Klunk, W. E., Koroshetz, W. J., Manly, J. J., Mayeux, R., Mohs, R. C., Morris, J. C., Rossor, M. N., Scheltens, P., Carrillo, M. C., Thies, B.,
Weintraub, S., and Phelps, C. H. (2011). The diagnosis of dementia due to Alzheimer's disease: recommendations from the National Institute on Aging-Alzheimer's Association workgroups on diagnostic guidelines for Alzheimer's disease. Alzheimers Dement. 7, 263-269.

Meulenbroek, O., Rijpkema, M., Kessels, R. P. C., Rikkert, M. G. M. O., and Fernández, G. (2010). Autobiographical memory retrieval in patients with Alzheimer's disease. Neuroimage 53, 331-340.

Mevel, K., Chételat, G., Eustache, F., and Desgranges, B. (2011). The default mode network in healthy aging and Alzheimer's disease. Int. J. Alzheimers Dis. 2011, 535816.

Mevel, K., Desgranges, B., Baron, J.C., Landeau, B., de La Sayette, V., Viader, F., Eustache, F., and Chételat, G. (2007). Detecting hippocampal hypometabolism in mild cognitive impairment using automatic voxelbased approaches. Neuroimage 37, 18-25.

Pariente, J., Cole, S., Henson, R., Clare, L., Kennedy, A., Rossor, M., Cipoloti, L., Puel, M., Demonet, J. F., Chollet, F., and Frackowiak, R. S. (2005). Alzheimer's patients engage an alternative network during a memory task. Ann. Neurol. 58, 870-879.

Persson, J., Lind, J., Larsson, A., Ingvar, M., Sleegers, K., Van Broeckhoven, C., Adolfsson, R., Nilsson, L.-G., and Nyberg, L. (2008). Altered deactivation in individuals with genetic risk for Alzheimer's disease. Neuropsychologia 46, 1679-1687.

Petrella, J. R., Wang, L., Krishnan, S., Slavin, M. J., Prince, S. E., Tran, T.T. T., and Doraiswamy, P. M. (2007). Cortical deactivation in mild cognitive impairment: high-field-strength functional MR imaging. Radiology 245, 224-235.

Pihlajamäki, M., DePeau, K. M., Blacker, D., and Sperling, R. A. (2008). Impaired medial temporal repetition suppression is related to failure of parietal deactivation in Alzheimer disease. Am. J. Geriatr. Psychiatry 16, 283-292.

Pihlajamäki, M., O’ Keefe, K., Bertram, L., Tanzi, R. E., Dickerson, B. C., Blacker, D., Albert, M. S., and Sperling, R. A. (2010). Evidence of altered posteromedial cortical FMRI activity in subjects at risk for Alzheimer disease. Alzheimer Dis. Assoc. Disord. 24, 28-36.

Rémy, F., Mirrashed, F., Campbell, B., and Richter, W. (2005). Verbal episodic memory impairment in Alzheimer's disease: a combined structural and functional MRI study. Neuroimage 25, 253-266.

Rombouts, S. A. R. B., Barkhof, F., Goekoop, R., Stam, C. J., and Scheltens, P. (2005). Altered resting state networks in mild cognitive impairment and mild Alzheimer's disease: an fMRI study. Hum. Brain Mapp. 26, 231-239.

Salmon, E., Lekeu, F., Bastin, C., Garraux, G., and Collette, F. (2008). Functional imaging of cognition in Alzheimer's disease using positron emission tomography. Neuropsychologia 46, 1613-1623.

Schröder, J., Buchsbaum, M. S., Shihabuddin, L., Tang, C., Wei, T. C., Spiegel-Cohen, J., Hazlett, E. A., Abel, L., Luu-Hsia, C., Ciaravolo, T. M., Marin, D., and Davis, K. L. (2001). Patterns of cortical activity and memory performance in Alzheimer's disease. Biol. Psychiatry 49, 426-436.

Schwindt, G. C., and Black, S. E. (2009). Functional imaging studies of episodic memory in Alzheimer's disease: a quantitative meta-analysis. Neuroimage 45, 181-190.

Solé-Padullés, C., Bartrés-Faz, D., Junqué, C., Vendrell, P., Rami, L., Clemente, I. C., Bosch, B., Villar, A., Bargalló, N., Jurado, M. A., Barrios, M., and Molinuevo, J. L. (2009). Brain structure and function related to cognitive reserve variables in normal aging, mild cognitive impairment and Alzheimer's disease. Neurobiol. Aging 30, 1114-1124.

Spaniol, J., Davidson, P. S. R., Kim, A S. N., Han, H., Moscovitch, M., and Grady, C. L. (2009). Event-related fMRI studies of episodic encoding and retrieval: meta-analyses using activation likelihood estimation. Neuropsychologia 47, 1765-1779.

Sperling, R. (2007). Functional MRI studies of associative encoding in normal aging, mild cognitive impairment, and Alzheimer's disease. Ann. N. Y. Acad. Sci. 1097, 146-155.

Sperling, R. A., Bates, J. F., Chua, E. F., Cocchiarella, A. J., Rentz, D. M., Rosen, B. R., Schacter, D. L., and Albert, M. S. (2003). fMRI studies of associative encoding in young and elderly controls and mild Alzheimer's disease. J. Neurol. Neurosurg. Psychiatr. 74, 44-50.

Sperling, R. A., Dickerson, B. C., Pihlajamaki, M., Vannini, P., LaViolette, P. S., Vitolo, O. V., Hedden, T., Becker, J. A., Rentz, D. M., Selkoe, D. J., and Johnson, K. A. (2010). Functional alterations in memory networks in early Alzheimer's disease. Neuromol. Med. 12, 27-43.

Stern, Y., Moeller, J. R., Anderson, K. E., Luber, B., Zubin, N. R., DiMauro, A. A., Park, A., Campbell, C. E., Marder, K., Bell, K., Van Heertum, R., and Sackeim, H. A. (2000). Different brain networks mediate task performance in normal aging and $\mathrm{AD}$ : defining compensation. Neurology 55, 1291-1297.

Tulving, E. (1995). “Organization of memory: quo vadis?" in The Cognitive Neurosciences, ed. M. Gazzaniga (Cambridge MA: The MIT Press), 839-847.

Viard, A., Piolino, P., Desgranges, B., Chételat, G., Lebreton, K., Landeau, B., Young, A., De La Sayette, V., and Eustache, F. (2007). Hippocampal activation for autobiographical memories over the entire lifetime in healthy aged subjects: an fMRI study. Cereb. Cortex 17, 2453-2467.

Villain, N., Desgranges, B., Viader, F., de La Sayette, V., Mézenge, F., Landeau, B., Baron, J.-C., Eustache, F., and Chételat, G. (2008). Relationships between hippocampal atrophy, white matter disruption, and gray matter hypometabolism in Alzheimer's disease. J. Neurosci. 28, 6174-6181.

Conflict of Interest Statement: The authors declare that the research was conducted in the absence of any commercial or financial relationships that could be construed as a potential conflict of interest.

Received: 22 December 2011; paper pending published: 07 February 2012; accepted: 10 April 2012; published online: 01 May 2012.

Citation: Bejanin A, Viard A, Chételat $G$, Clarys D, Bernard F, Pélerin $A$, de La Sayette $V$, Eustache $F$ and Desgranges $B$ (2012) When higher activations reflect lower deactivations: a PET study in Alzheimer's disease during encoding and retrieval in episodic memory. Front. Hum. Neurosci. 6:107. doi 10.3389/fnhum.2012.00107

Copyright $(\odot 2012$ Bejanin, Viard, Chételat, Clarys, Bernard, Pélerin, de La Sayette, Eustache and Desgranges. This is an open-access article distributed under the terms of the Creative Commons Attribution Non Commercial License, which permits non-commercial use, distribution, and reproduction in other forums, provided the original authors and source are credited. 